PEG and 15\% RIG. The mean insertion time from diagnosis was 11 months (+/- 14 months), with 30 day mortality $1.9 \%$. Overall mortality was $87 \%$ with mean survival from diagnosis 23 months (+/- 16 months).

The majority of PEG insertions were arranged on dedicated lists with anaesthetic cover ( $83 \%$ vs $17 \%$ ) compared with only a minority of RIG insertions (18\% vs $82 \%)$. There was no significant difference between time to insertion $(\mathrm{P}=0.78)$ and survival from diagnosis $(\mathrm{P}=0.61)$ between the 2 cohorts.

Conclusions Gastrostomy use in the South West is safe, with mortality rates below quoted literature. Gastrostomy practice differs between the 2 hospitals in the approach used but time to insertion and mean survival was not different. The authors intend to pursue a joint referral pathway for gastrostomy assessment. This would improve data collection quality, allowing future analysis of standardised variables to ascertain the most effective use of gastrostomies in these patients.

\section{PWE-006 THE T-PIECE PULL TECHNIQUE REMOVAL OF PEG BURIED BUMPERS - A QUICK NOVEL ENDOSCOPIC TECHNIQUE}

Thomas Hollingworth*, Philip Boger, E Clarke, P Patel, C Rutter, T Smith, Imdadur Rahman. University Hospital Southampton, Southampton, UK

\subsection{6/gutjnl-2019-BSGAbstracts.337}

Introduction Buried bumper syndrome (BBS) represents a rare, but potentially serious complication in percutaneous endoscopic gastrostomy (PEG) tubes. In BBS there is a growth of the gastric mucosa over the inner bumper of the PEG tube, affecting $0 .-\%$ of PEG tubes.

Options for the management of BBS include the insertion of a jejunal extension, surgical or endoscopic removal. Endoscopic removal represents the most attractive option as it is the least invasive procedure to remove the buried bumper (BB). Several endoscopic techniques have been described including endoscopic dissection (ESD) of the overgrown tissue, a "Push-pull T technique", or the use of a papillotome placed through the PEG tube to cut the overgrown tissue.

The novel T-piece pull technique has been developed as a modification of the "Push-pull $\mathrm{T}$ technique" without the need for external support.

Methods A prospectively kept database was analysed for all BBS patients undergoing the procedure between September 2016 to January 2019. Primary outcome measures were technical success, procedural times and adverse events. Secondary outcome measures were BB morphology based on Paris polyp classification and PEG dwell time.

Description of technique (Figure 1)

1. Following endoscopic assessment of the BB, the PEG tube is exposed and cleaned.

2. The PEG tube is cut close to the abdominal wall.

3. Biopsy forceps are passed through the PEG tube into the stomach under direct endoscopic vision.

4. The forceps pull a $10 \mathrm{~mm}$ snare passed down the biopsy channel of endoscope out of the stomach through the PEG tube.

5. A $2 \mathrm{~cm}$ piece of the redundant PEG tube is cut and placed through the snare and closed to create a t-piece.

6. The snare is pulled firmly through the PEG into the stomach, removing the buried bumper.

Results A total of 27 cases for BBS were undertaken with the T-Piece pull technique, on 18 females and 9 males with a mean age of 43 (range 1-8). The median PEG tubes dwell time was 14 months. Procedures were performed with conscious sedation in $19 \%$ and enhanced sedation with anaesthetic support in $81 \%$. BB morphology was Paris 1s in 9, 2a in 16 and $2 \mathrm{~b}$ in 2 . The median procedure time was 18 minutes (range 1-5). The technical success was 93\%. 2 failed procedures required surgical removal, both $2 \mathrm{~b}$ lesions. Complications occurred in 2 cases, 1 episode of sepsis and 1 abdominal pain.

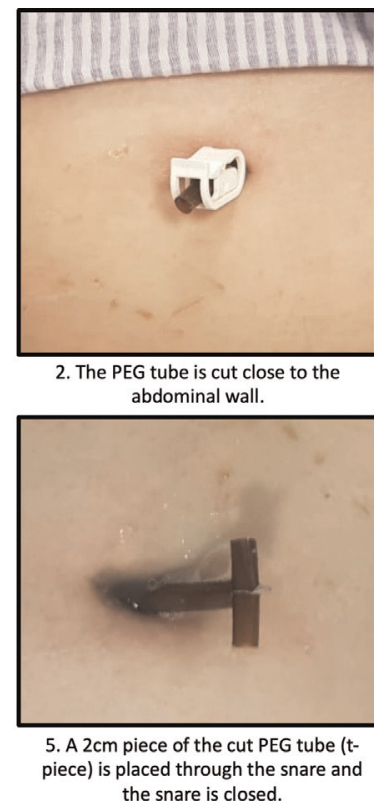
the snare is closed.

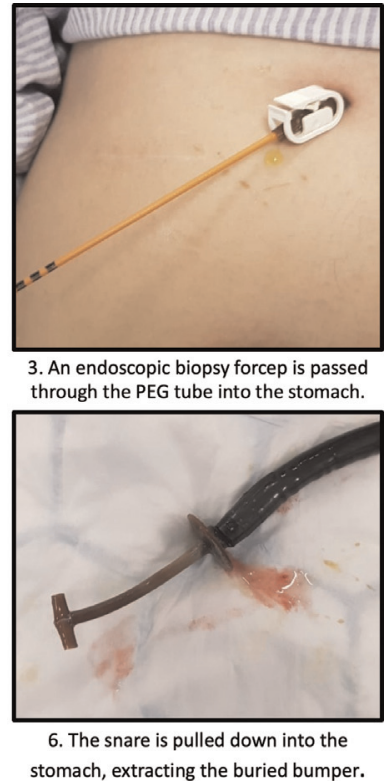


Discussion This novel T-piece pull technique for the removal of $\mathrm{BB}$ is a simple endoscopic procedure, using equipment that is readily available in most endoscopy departments. The procedure time is much shorter than more complex endoscopic techniques, such as ESD. Lesions which have a $2 \mathrm{~b}$ Paris classification indicate a deeply buried bumper and should be considered for a different approach to removal.

\section{PWE-007 TYPE 2 INTESTINAL FAILURE- A LARGE TERTIARY HOSPITAL EXPERIENCE}

Thomas Hollingworth*, Lucy Bakewell, Elizabeth Buse, Emily Clarke, Zillah Leach, Priya Mistry, Carl Richardson, Charlotte Rutter, Andrew King, Trevor Smith. University Hospital Southampton, Southampton, UK

\subsection{6/gutjnl-2019-BSGAbstracts.338}

Introduction Type 2 intestinal failure (IF) is a prolonged ( $>28$ days), acute condition requiring complex multi-disciplinary (MDT) care and intravenous (IV) supplementation, either in hospital or through the provision of home parenteral nutrition (HPN). Without careful MDT management, these patients can progress to type $3 \mathrm{IF}$, requiring lifelong IV support with associated morbidity. NHS England estimates that there will be an increase in the provision of HPN at a rate of approximately $20 \%$ per annum, largely as a consequence of the increase in type 2 IF.

The aim of this study was to assess the efficacy of IF surgery that we provide in our tertiary referral centre in reducing the need for ongoing IV support. Secondary end points included the reason that IV support was required, the length of time from commencing IV support to surgery and the underlying pathology that led to the IF.

Methods A prospectively kept database of all patients receiving HPN who underwent IF surgery between September 2009 and July 2018. The notes of these patients were assessed with the dates or referral, length of IV support, reason for IV support, underlying pathology and IV support required following surgery recorded.

Results 77 operations were performed on 76 patients with a median age at the time of operation of 57 (range 1-1). 64 patients received HPN whilst 12 received IV fluids. These patients required IV support prior to an IF laparotomy due to fistulae (31), high output stoma (26), abdominal sepsis (15), and bowel obstruction (5). The underlying pathology that led to their presentation includes inflammatory bowel disease (26), vascular (13), surgical complications (10), malignancy (7), diverticular disease (7), Volvulus (4), Radiotherapy (3), adhesions (2), perforation (2), pancreatitis, TB and in one case unknown. The median time from starting IV support to the date of surgery was 218 days (range 10-67) Of these 92\% were able to stop their HPN and $75 \%$ had their home IV fluids stopped. 5 patients had their TPN reduced to intravenous fluids and 8 continued with their previous home intravenous support

Conclusions Surgery for type $2 \mathrm{IF}$, in selected patients who have close MDT management can lead to a significant reduction in the requirement for home IV support, reducing the associated morbidity and mortality. The MDT needs to closely support patients nutritionally, psychologically and physically, allowing time for recovery from the initial insult before embarking on further surgery.

\section{PWE-008 DOSING, DURABILITY OF HAEMOGLOBIN RESPONSE AND SAFETY OF IRON ISOMALTOSIDE IN PATIENTS WITH GASTROINTESTINAL DISEASES}

Amanda Thomas, Vida Cairnes, Sean Mole, Claire Elworthy, Juan Saucedo Figueredo, James R Goodhand, Tariq Ahmad, Nicholas A Kennedy*. IBD Service, Royal Devon and Exeter NHS Foundation Trust, Exeter, UK

\subsection{6/gutjnl-2019-BSGAbstracts.339}

Introduction Iron deficiency anaemia (IDA) commonly complicates gastrointestinal disease and impairs quality of life (QoL). Intravenous iron therapy is widely used in IDA when oral iron is poorly tolerated or ineffective, and normalisation of haemoglobin improves QoL scores. We sought to define the dosing regimens of, durability of haemoglobin responses and the prevalence of adverse events to intravenous iron (III) isomaltoside (Monofer).

Methods We undertook a service evaluation of intravenous iron use in 508 outpatients (40\% male) with gastrointestinal disease treated with 648 Monofer infusions between 2014 and 2017. Demographic, diagnosis, and treatment factors including dose of Monofer used and the number of patients treated with repeat infusions were recorded form the medical record. Anaemia was defined by WHO criteria. Iron deficiency was defined as transferrin saturation $<18 \%$ and/or ferritin $<30$ $\mu \mathrm{g} / \mathrm{L}$ (ferritin $<100 \mu \mathrm{g} / \mathrm{L}$ if CRP $>5 \mathrm{mg} / \mathrm{L}$ ). We extracted laboratory results from the electronic record for baseline, 12 weeks and 52 weeks. We sought factors associated with treatment failure, defined as ongoing anaemia at 12 weeks, using logistic regression.

Results Overall, 93\% (568/613) were anaemic at baseline with median (IQR) haemoglobin of $100 \mathrm{~g} / \mathrm{L}$ (IQR 8-12). 73\% (476/ 648) had haematinics tested and 91\% [431/476] had proven IDA. Inflamatory bowel disease was the most common indication at $30 \%(193 / 648)$ infusions. A variety of dosing regimens were used: $26 \%(167 / 648)$ received fixed dosing of $1 \mathrm{~g}, 25 \%(163 /$ 648) were dosed according to the Ganzoni formula, 14\% (91/ 648) had the dose calculated by the Ganzoni formula but limited to a single $20 \mathrm{mg} / \mathrm{kg}$ infusion, $8 \%(54 / 648)$ were dosed by the simplified dosing table and $27 \%(173 / 648)$ other dosing strategies. 74\% (479/648) infusions had follow-up haemoglobin measured 6 to 18 weeks post-infusion. The median change in haemoglobin between baseline and -8 weeks was $18 \mathrm{~g} / \mathrm{L}$ (IQR 9). 42\% (186/438) of previously anaemic patients had normalised their haemoglobin by this time. Factors associated with failure to normalise haemoglobin were male sex (odds ratio (OR) 2.9 [95\%CI 1.-.3]), age $\geq 65$ years (OR 3.4 [95\%CI 2.-.0]), higher comorbidity (OR 3.7 [95\%CI 2.-.5]) and under-dosing versus Ganzoni-calculated dose (OR 4.8 per gram underdosed [95\%CI 2.-0]). Only, 31\% (44/143) patients whose haemoglobin normalised at week 12 had recurrent anaemia at 1 year. Adverse events were rare: only one patient had a probable complement activation-related pseudoallergy that was mitigated by slowing infusions, and there were no anaphylactic reactions.

Discussion A wide-variety of dosing strategies are used in our trust. Treatment failure was associated with under-dosing , age, sex and comorbidity. Adverse events were rare. 